\title{
An integrated analysis of cancer genes in thyroid cancer
}

\author{
$\mathrm{LI} \mathrm{CHAI}^{1}, \mathrm{JIA} \mathrm{LI}^{2}$ and $\mathrm{ZHONGWEI} \mathrm{LV}{ }^{1}$ \\ Departments of ${ }^{1}$ Nuclear Medicine and ${ }^{2}$ Thyroid and Breast, Shanghai Tenth People's Hospital, \\ Tongji University, School of Medicine, Shanghai 200072, P.R. China
}

Received September 5, 2015; Accepted October 16, 2015

DOI: 10.3892/or.2015.4466

\begin{abstract}
Cancer driver genes are commonly mutationally disrupted in cancer, which confers a growth advantage to tumor cells. Recent studies preferentially search for recurrently mutated driver genes across multiple tumor samples, leading to the neglect of low-frequency mutated cancer genes. The present study was conducted to identify cancer-driving genes in thyroid cancer with two distinct tools, OncodriveFM and Dendrix, which aim to detect neglected driver genes with low mutation frequency. A total of 23,620 somatic mutations generated by whole-exome sequencing of 446 tumor/normal pairs of thyroid cancer were obtained from TCGA. Variant classification was conducted with Ensembl Variant Effect Predictor (VEP). OncodriveFM and Dendrix were applied to detect driver genes and pathways with statistical evidence. In addition, we analyzed DNA-methylation status, copy number variation, expression levels and fusion genes among these driver candidates. In total, non-synonymous mutations accounted for over $55 \%(13,091 / 23,620)$ of the total variants; 53 and 3 driver genes were determined by OncodriveFM and Dendrix, respectively, including 6 recurrently mutated driver genes, such as BRAF, NRAS, HRAS, EIF1AX, KRAS and 47 new genes. A total of 75 pathways with high function impact bias were identified by OncodriveFM. Two genes, FHOD3 and SRP72, were hypomethylated, overexpressed and involved in major deletions in thyroid cancer. Moreover, we identified 91 pairs of fusion genes, 89 of which were new fusion pairs in thyroid cancer. In conclusion, we successfully identified a list of new cancer genes, pathways and fusion genes, providing better insight into the tumorigenesis of thyroid cancer.
\end{abstract}

\section{Introduction}

Over the past decade, the incidence rate of thyroid cancer has greatly increased (1). Thyroid cancer can be histologically classified into many subtypes, including follicular, anaplastic,

Correspondence to: Dr Zhongwei Lv, Department of Nuclear Medicine,Shanghai Tenth People's Hospital,Tongji University, School of Medicine, 301 Yanchang Road, Shanghai 200072, P.R. China E-mail: doctorc1@163.com

Key words: thyroid cancer, driver mutation, driver gene, driver pathway, fusion gene medullary and papillary thyroid cancer (PTC), among which PTC is the most prevalent. PTCs usually have a good survival and prognosis with a 5-year survival rate higher than $95 \%$ (2); however, these cancers occasionally become aggressive and deadly through dedifferentiation into other subtypes, such as dedifferentiated thyroid cancer. Current treatments include surgery, thyroid hormone and radioactive iodine (RAI) therapy.

Identification of cancer-driving genes has been consistently a hotspot in cancer genomic research, and to date, 547 cancer genes are annotated in the COSMIC database (3). The common approach to detect driver searches for genes that are significantly mutated in a cohort of cancer samples as compared to the background mutation rate, is through MutSigCV (4) and MuSiC (5). Application of MutSig to 496 paired tumor/normal samples has found many over-mutated cancer drivers in thyroid cancer, such as BRAF, NRAS, HRAS, EIF1AX and KRAS (6). However, new evidence shows that many driver genes may occur at a low frequency; for example, some cancer drivers are mutated in a small fraction (e.g., <1\%) of tumors (7). Therefore, current tools may overlook potential drivers that are mutated at a low frequency in the cancer genome, and methods that could identify these low-frequency mutated driver genes are urgently needed. Methods such as OncodriveFM (8) tend to detect genes that have bias toward the accumulation of variants with high functional impact measured by SIFT (9), PolyPhen2 (10) and MutationAssessor (11). Another new method Dendrix based on mutual exclusivity was developed to find sets of genes in which the majority of cancer samples have at least one mutation, while display a mutation in one of the genes (12). These prediction tools complementary to existing methods provide new opportunities to identify cancer genes that drive tumor formation and progression.

In the present study, we describe the analysis of somatic mutations detected by whole exome sequencing of 446 normal/tumor pairs of thyroid cancer samples from the Cancer Genome Atlas (TCGA) database, OncodriveFM and Dendrix were applied to prioritize cancer driver genes and pathways. We identified 53 cancer driver candidates and 75 pathways with significant bias of functional impact. In addition, we analyzed DNA methylation status, copy number variation, expression levels and fusion genes among these driver candidates. We found that two genes, FHOD3 and SRP72, were hypomethylated, overexpressed and involved in major deletions, suggesting that they play an oncogenic role in thyroid cancer. Our study highlights the importance of identifying low-frequency mutated cancer-driving genes in an integrated way. 


\section{Materials and methods}

Prediction of the functional effect of cancer mutations, genes and pathways. A total of 23,620 somatic mutations detected by whole-exome sequencing of 446 tumor/normal pairs were obtained from TCGA (http://cancergenome.nih.gov/, download on July 14, 2015). Functional impact of somatic mutations in the coding genome was classified with Ensembl Variant Effect Predictor (VEP) (13). Cancer genes and pathways were predicted by OncodriveFM (8) and Dendrix (12) programs and all the parameters were set to default. Genes and pathways with Q-value $<0.05$ were regarded as cancer gene and pathway candidates. GO enrichment analysis was performed for all of the driver candidates from the home page of the GOC website (14) (http://geneontology.org/).

DNA methylation, RNA-seq data processing and expression analyses. DNA methylation data for 389 thyroid cancer samples were obtained from TCGA, and the undefined value was replaced with an average $\beta$ value. The average $\beta$ value was computed for each gene and cancer sample. RNA-seq data of 18 papillary thyroid carcinoma biopsies and 4 normal thyroid tissues were obtained from the study of Costa et al (GSE64912) (15). Read alignment with human genome 19 was conducted with TopHat2 (16), and read count was computed with bedtools v2.22.1 (17) for each gene. Differentially expressed genes were determined with DEseq2 (18) package in R between tumor and normal tissues with cutoffs of a false discovery rate (FDR) $\leq 0.5$ and absolute fold-change $\geq 2$.

Sources of copy number variation and fusion gene data. We obtained copy number variations of 501 thyroid cancer samples which were detected by SNP array and publically available at the Broad Institute (6) (gdac.broadinstitute.org). RNA-seq data from the study of Costa $e t$ al was aligned to the human genome with Tophat2, fusion genes were detected with TopHat-Fusion (16), and all of the parameters were set by default.

Statistical analysis. Data are presented as mean, and differences between different groups were drawn with the Wilcoxon rank sum test in $\mathrm{R} . \mathrm{P}<0.05$ was considered to indicate a statistically significant difference and the null hypothesis was rejected.

\section{Results}

Catalogue of somatic mutations. A total of 23,620 somatic mutations detected by whole-exome sequencing of 446 thyroid cancer specimens were obtained from TCGA. Among these, 23,070 were single-nucleotide variants ( $\mathrm{SNVs}$ ), 550 were small insertions or deletions. $\mathrm{C}>\mathrm{T} / \mathrm{G}>\mathrm{A}, \mathrm{C}>\mathrm{A} / \mathrm{G}>\mathrm{T}$ and $\mathrm{T}>\mathrm{C} / \mathrm{A}>\mathrm{G}$ accounted for $32.10,21.04$ and $19.21 \%$ of the variant types in the non-CpG sites, and $4.42,1.65$ and $1.35 \%$ of variant types in the $\mathrm{CpG}$ islands. $\mathrm{C}>\mathrm{T} / \mathrm{G}>\mathrm{A}, \mathrm{C}>\mathrm{A} / \mathrm{G}>\mathrm{T}$ and $\mathrm{T}>\mathrm{C} / \mathrm{A}>\mathrm{G}$ were the three predominant transitions in thyroid cancer (Fig. 1A). A total of 12,817, 274 and 5 single nucleotide variations were classified as missense, nonsense and nonstop mutations, respectively by VEP. A total of 7,988 single nucleotide varia- tions were classified as silent. A total of 318 and 103 small deletions and insertions introduced translational frameshifts, and 72 and 8 small deletions and insertions were in frame mutations. A total of 448 and 22 mutations were located in splicing sites and translation start sites, while non-synonymous mutations accounted for $>55 \%(13,091 / 23,620)$ of the total variants (Fig. 1B). Thyroid cancer showed a significantly lower non-synonymous mutation density $(0.31$ non-synonymous mutations per $\mathrm{Mb}$ per sample, on average) as compared to other cancers, such as melanoma and lung cancer $(4,19)$.

Cancer driver genes and pathways in thyroid cancer. We applied OncodriveFM to identify driver genes in thyroid cancer. In total, 53 genes were determined as driver candidates by OncodriveFM. Among them, BRAF, NRAS, HRAS, KRAS, PPM1D and EIF1AX are known recurrently mutated driver genes in thyroid cancer, with mutation frequencies of $59.64,8.52,3.59,1.12,1.35$ and $1.35 \%$ across all samples (6). However, most of the driver candidates were not frequently mutated in thyroid cancer (Fig. 2A and B). The P53 signaling genes were also determined as driver genes; TP53, ATM and CHEK2, showed relatively low mutation frequencies $(<2.5 \%$ in all cases), suggesting the inactivation of the P53 signaling pathway in thyroid cancer. Several known cancer genes of other cancer types were first identified as drivers in thyroid cancer, such as BRCA1 in breast cancer (20), MLL and MLL3 in hepatocellular carcinoma (HCC) (21) and gastric cancer (22), ATM in glioma (23) and leukemia (24), PTPN11 in acute myeloid leukemia (25) as well as DICER1 in pleuropulmonary blastoma (26). In addition, we also identified many new driver candidates, for example, mitogen-activated protein kinase kinase kinase 3 (MEKK3) and Transport and Golgi organisation protein 1 (TANGO). MEKK3 is elevated in esophageal squamous cell carcinoma (ESCC), and overexpression of MEKK3 indicates poor prognosis of ESCC (27). Breast and ovarian cancers show an elevated MEKK3 protein level and increased NF- $\kappa \mathrm{B}$ binding activity, and overexpression of MEKK 3 was found to activate NF- $\kappa B$ binding activity and upregulate cell survival and anti-apoptotic genes such as Bcl-2 and XIAP in U373 cells, which enhanced cellular resistance to apoptosis induced by chemotherapeutic agents (28). Another driver candidate, MIA3, also known as TANGO, is a member of the melanoma inhibitory activity (MIA) gene family. It displays a tumor-suppressor function in multiple cancer types, such as human colon and hepatocellular carcinoma (29) and melanoma (30).

Dendrix was developed to identify sets of genes which are mutated in a large fraction of cancer samples and whose mutations are mutually exclusive. We next analyzed somatic mutations of thyroid cancer with Dendrix. In total, 10,839 genes were reported as mutated in at least one patient. We performed Dendrix for sets with sizes ranging from 2 to 5 . When $\mathrm{k}=2$, the pair BRAF and NRAS was sampled $96.1 \%$ of the time. When $\mathrm{k}=3$, the triplet (BRAF, NRAS and KRAS) was sampled with a frequency of $22.2 \%$. For $k=4$, no gene set had sample frequency $>1 \%$. The pair (BRAF, NRAS) and triplet (BRAF, NRAS and KRAS) were the most prevalent gene sets in the mutual exclusivity test, further supporting the importance of BRAF, NRAS and KRAS in the tumorigenesis of thyroid cancer. 
A

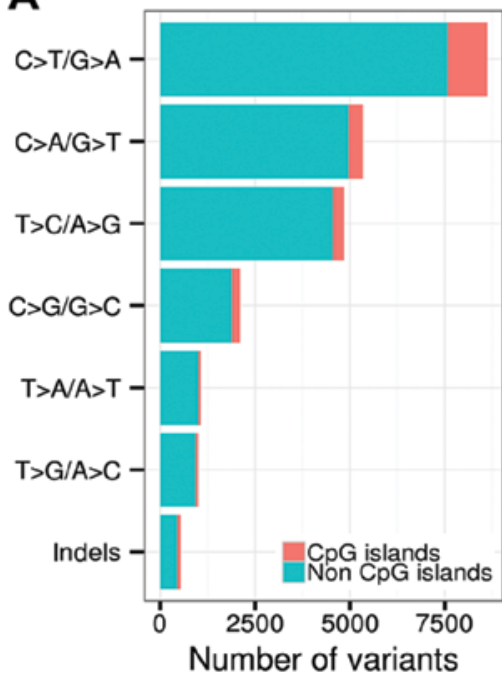

B

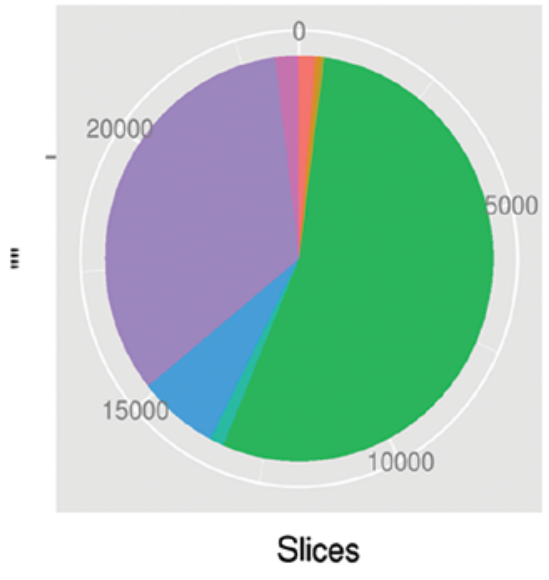

Ibls

Frame_Shift_Del $1 \% 1.35 \%$

Frame-Shift Ins $0 \% 0.44 \%$

In_Frame_Dèl $0 \% 0.3 \%$

In Frame-Ins 0\% 0.03\%

Missense-Mutation $54 \% 54.26 \%$

Nonsense Mutation $1 \% 1.16 \%$

Nonstop Mutation 0\% $0.02 \%$

RNA $7 \% 6.63 \%$

Silent $34 \% 33.82 \%$

Splice Site 2\% 1.9\%

ranslation_Start_Site 0\% 0.09\%

Figure 1. (A) Mutation signatures in thyroid cancer; (B) the number and proportion of mutation classes with different functional impact in thyroid cancer.

A

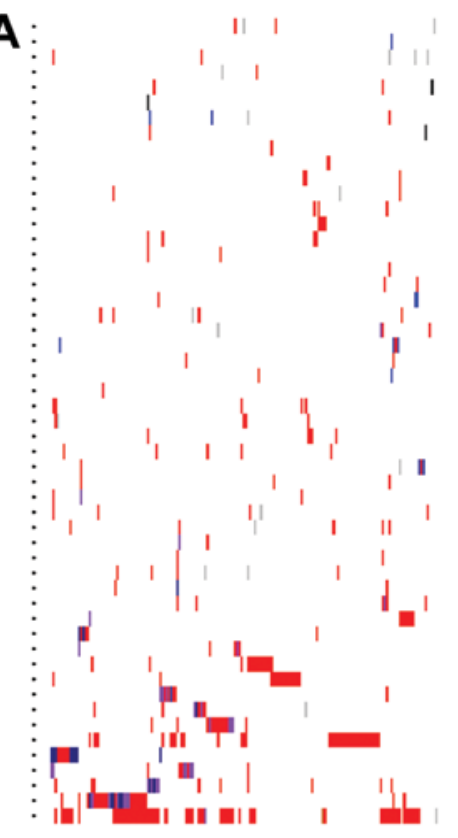

B

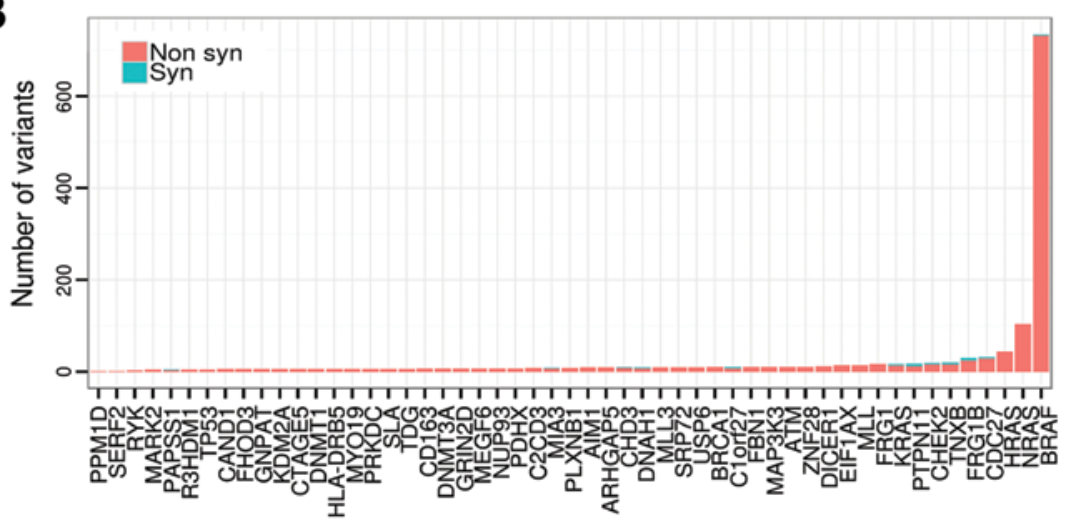

Figure 2. (A) Gene-sample matrix of mutations across all driver candidates; (B) the number of synonymous (syn) and non-synonymous (non-syn) mutations in all driver candidates.

We analyzed the enrichment of GO terms of the 53 cancer gene candidates, and $27 \mathrm{GO}$ terms were determined with

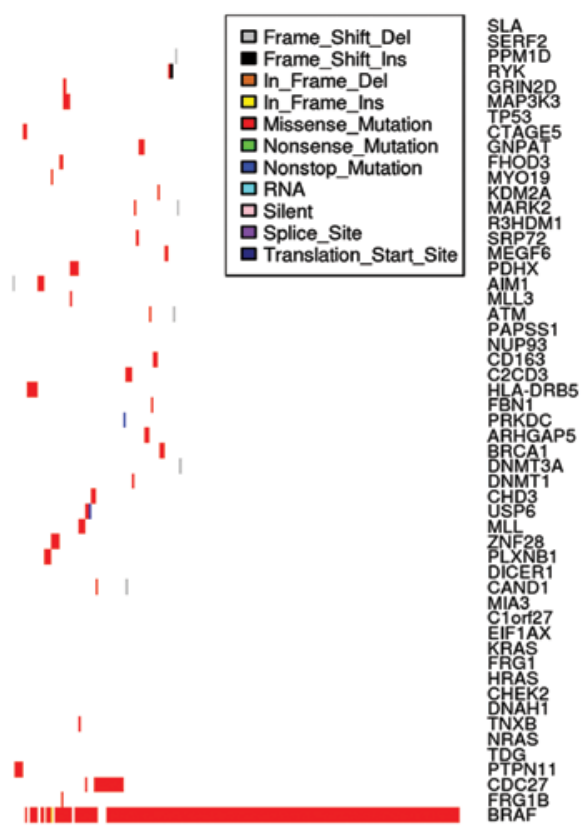

| 
Table I. Enrichment of GO terms for driver candidates in thyroid cancer.

\begin{tabular}{|c|c|c|c|}
\hline GO terms & No. of genes & Fold-change of enrichment & P-value \\
\hline Replicative senescence & 3 & $>5$ & $2.21 \mathrm{e}-02$ \\
\hline Intrinsic apoptotic signaling pathway in response to DNA damage & 5 & $>5$ & $8.74 \mathrm{e}-03$ \\
\hline Signal transduction in response to DNA damage & 5 & $>5$ & $4.46 \mathrm{e}-02$ \\
\hline Response to ionizing radiation & 6 & $>5$ & $6.61 \mathrm{e}-03$ \\
\hline Double-strand break repair & 6 & $>5$ & $6.90 \mathrm{e}-03$ \\
\hline DNA damage checkpoint & 6 & $>5$ & $1.09 \mathrm{e}-02$ \\
\hline DNA integrity checkpoint & 6 & $>5$ & $1.59 \mathrm{e}-02$ \\
\hline Mitotic cell cycle checkpoint & 6 & $>5$ & $2.44 \mathrm{e}-02$ \\
\hline Intrinsic apoptotic signaling pathway & 6 & $>5$ & $3.08 \mathrm{e}-02$ \\
\hline Cell cycle checkpoint & 8 & $>5$ & $2.06 \mathrm{e}-03$ \\
\hline Response to radiation & 9 & $>5$ & $1.18 \mathrm{e}-02$ \\
\hline Cellular response to nitrogen compound & 12 & $>5$ & $3.06 \mathrm{e}-04$ \\
\hline Cellular response to organonitrogen compound & 11 & $>5$ & $1.38 \mathrm{e}-03$ \\
\hline Cell cycle process & 13 & $>5$ & $7.82 \mathrm{e}-03$ \\
\hline Response to nitrogen compound & 12 & $>5$ & $2.42 \mathrm{e}-02$ \\
\hline Positive regulation of protein modification process & 13 & 4.73 & $1.68 \mathrm{e}-02$ \\
\hline Cellular component morphogenesis & 13 & 4.54 & $2.61 \mathrm{e}-02$ \\
\hline Cell cycle & 14 & 4.41 & $1.42 \mathrm{e}-02$ \\
\hline Regulation of protein modification process & 16 & 3.97 & $8.52 \mathrm{e}-03$ \\
\hline Macromolecular complex subunit organization & 17 & 3.41 & $2.82 \mathrm{e}-02$ \\
\hline Macromolecule modification & 22 & 3.13 & $2.26 \mathrm{e}-03$ \\
\hline Regulation of protein metabolic process & 19 & 3.13 & $2.20 \mathrm{e}-02$ \\
\hline Cellular protein modification process & 20 & 2.99 & $2.10 \mathrm{e}-02$ \\
\hline Protein modification process & 20 & 2.99 & $2.10 \mathrm{e}-02$ \\
\hline Cellular component organization or biogenesis & 33 & 2.65 & $8.59 \mathrm{e}-06$ \\
\hline Cellular component organization & 32 & 2.63 & $2.58 \mathrm{e}-05$ \\
\hline Response to stress & 23 & 2.62 & $2.43 \mathrm{e}-02$ \\
\hline
\end{tabular}

pathway, regulation of protein metabolic process and cell cycle and DNA damage checkpoint. These findings further support that the driver candidates identified by OncodriveFM and Dendrix have critical functions in thyroid cancer. OncodriveFM also revealed 75 pathways with high FM bias in thyroid cancer, such as regulation of actin cytoskeleton, melanoma, thyroid cancer, renal cell carcinoma, bladder cancer, non-small cell lung cancer (NSCLC), alcoholism, endometrial cancer, prostate cancer, glioma, chronic myeloid leukemia and acute myeloid leukemia (Table II).

DNA methylation in thyroid cancer. Epigenetic alterations such as methylation of cytosine-guanine dinucleotides $(\mathrm{CpG})$ play an important role in human carcinogenesis. We obtained DNA methylation data from TCGA and analyzed its association with driver genes in thyroid cancer. First of all, we used hierarchical clustering to analyze DNA methylation profiling and found two clusters of hypermethylated and hypomethylated genes (Fig. 3A). This suggested that genes with hypermethylation or hypomethylation on promoters are associated with tumor suppressors or oncogenes (31). We selected the top 500 hypermethylated and hypomethylated genes which had the highest or lowest mean $\beta$ value, respectively, and analyzed functional enrichment with GO terms.
Hypomethylated genes were significantly enriched in $80 \mathrm{GO}$ terms. Among them, many were found to be associated with cell cycle regulation, metabolic process, cell division and gene expression, while, hypermethylated genes were enriched in only in $9 \mathrm{GO}$ terms, such as detection of stimulus, G-protein coupled receptor signaling pathway and sensory perception of smell. In addition, we obtained RNA-seq data of 18 papillary thyroid carcinoma biopsies and 4 normal thyroid tissues from the study of Costa et al (15). We analyzed the expression levels of hypermethylated and hypomethylated genes. The hypermethylated genes were found to be significantly lower expressed as compared to the hypomethylated ones (RPKM, 3.08 vs. $4.72, \mathrm{P}<2.2 \mathrm{e}-16$; Wilcoxon rank sum test) (Fig. 3B). Among all the driver candidates, CD163, USP6, R3HDM1, DNAH1, ARHGAP5 and R3HDM1 showed hypermethylation, while PPM1D, SRP72, GNPAT and TDG exhibited hypomethylation (Fig. 3C), suggesting they may be involved in tumorigenesis of thyroid cancer by altering the methylation status. We also identified 504 differentially expressed genes between thyroid cancer and normal tissues (Fig. 4A). Among them, four were driver gene candidates, including AIM1, FHOD3, SRP72 and FRG1B (Fig. 4B). AIM1, SRP72 and FRG1B are overexpressed and hypomethylated, which suggests they may have oncogenic function in thyroid cancer. 
Table II. Top 20 cancer-driving pathways detected by OncodriveFM in thyroid cancer.

\begin{tabular}{lcccc}
\hline Pathway name & Pathway ID & Gene number & P-value & Q-value \\
\hline Regulation of actin cytoskeleton & hsa04810 & 213 & $1.10 \mathrm{e}-182$ & $1.39 \mathrm{e}-181$ \\
Chemokine signaling pathway & hsa04062 & 189 & $8.79 \mathrm{e}-178$ & $1.06 \mathrm{e}-176$ \\
Natural killer cell mediated cytotoxicity & hsa04650 & 132 & $1.11 \mathrm{e}-174$ & $1.27 \mathrm{e}-173$ \\
Hepatitis C & hsa05160 & 131 & $9.96 \mathrm{e}-184$ & $1.42 \mathrm{e}-182$ \\
Melanoma & hsa05218 & 71 & $3.11 \mathrm{e}-213$ & $2.37 \mathrm{e}-211$ \\
Thyroid cancer & hsa05216 & 29 & $1.80 \mathrm{e}-213$ & $2.05 \mathrm{e}-211$ \\
Renal cell carcinoma & hsa05211 & 70 & $7.99 \mathrm{e}-219$ & $1.82 \mathrm{e}-216$ \\
Long-term potentiation & hsa04720 & 70 & $9.00 \mathrm{e}-190$ & $1.71 \mathrm{e}-188$ \\
ErbB signaling pathway & hsa04012 & 88 & $1.50 \mathrm{e}-183$ & $2.02 \mathrm{e}-182$ \\
Neurotrophin signaling pathway & hsa04722 & 119 & $8.30 \mathrm{e}-190$ & $1.71 \mathrm{e}-188$ \\
Serotonergic synapse & hsa04726 & 112 & $1.40 \mathrm{e}-193$ & $3.54 \mathrm{e}-192$ \\
Alcoholism & hsa05034 & 177 & $1.02 \mathrm{e}-206$ & $5.80 \mathrm{e}-205$ \\
Glioma & hsa05214 & 65 & $1.67 \mathrm{e}-197$ & $5.45 \mathrm{e}-196$ \\
Non-small cell lung cancer & hsa05223 & 54 & $1.56 \mathrm{e}-197$ & $5.45 \mathrm{e}-196$ \\
Bladder cancer & hsa05219 & 42 & $1.39 \mathrm{e}-204$ & $6.34 \mathrm{e}-203$ \\
Endometrial cancer & hsa05213 & 52 & $1.89 \mathrm{e}-192$ & $4.31 \mathrm{e}-191$ \\
Prostate cancer & hsa05215 & 88 & $1.94 \mathrm{e}-189$ & $3.40 \mathrm{e}-188$ \\
Chronic myeloid leukemia & hsa05220 & 73 & $9.45 \mathrm{e}-196$ & $2.69 \mathrm{e}-194$ \\
Long-term depression & hsa04730 & 61 & $9.04 \mathrm{e}-187$ & $1.47 \mathrm{e}-185$ \\
Acute myeloid leukemia & hsa05221 & 57 & $1.69 \mathrm{e}-185$ & $2.57 \mathrm{e}-184$ \\
\hline
\end{tabular}

Copy number variations in thyroid cancer. We also obtained copy number variations of 501 thyroid cancer samples detected by the Broad Institute. Significant focal gains and deletions $(\mathrm{Q}<0.25)$ were found in 247 samples $(247 / 501,49.30 \%)$ at 107 loci (68 amplifications and 39 deletions). Among them, deletions at $2 \mathrm{q} 13.32,22 \mathrm{q} 13.2,22 \mathrm{q} 13.1$ and $22 \mathrm{q} 12.3$ were the most frequent copy number variations in thyroid cancer, with an occurrence rate of 17.96 (90/501), 17.76 (89/501), 17.76 $(89 / 501)$ and $17.96 \%$ (90/501), respectively (Fig. 5). Several known tumor suppressors and oncogenes were involved in copy number variation, including BRAF (amplification and deletion, 7q34), TP53 and BRCA1 (deletion, 17p13.1), EIF1AX (amplification and deletion, Xp22.12 and Xq23). Many driver candidates were also found to be implicated in the CNVs, including FRG1, PAPSS1 and SRP72 (deletion, 4q22.3), CDC27, CDH3, PPM1D, USP6, MYO19, (17p13.1), DNMT3A and R3HDM1 (2q14.3) and FHOD3 (18q12.3), NUP93(16p13.3) DNMT1 (19p13.2).

Fusion genes in thyroid cancer. We obtained RNA-seq data of 18 papillary thyroid carcinoma samples from the study of Costa et al (15), and applied Tophat-Fusion to detect potential fusiongenes in thyroid cancer.Intotal, we found 91 pairs of fusion genes in 18 cancer samples (Fig. 6A). C7orf50-LOC101927021, ATG7-VGLL4, RP11-131L23.1-ZNHIT6, MRPS28-TPD52 and SLC28A3-FRMD3 were among the most frequent fusion pairs in thyorid cancer, with a frequency of $94.44,88.89$, $88.89,77.78$ and $77.78 \%$ in the cancer samples (Fig. 6B). We compared our list of fusion gene pairs with known fusion genes of thyroid cancer from TCGA Fusion Gene Data Portal (http://54.84.12.177/PanCanFusV2/). There were only two known fusion gene pairs, ANK3-RET (33.33\%) and PPARG-PAX8 (5.56\%), while the majority of pairs of fusion genes were newly identified. In addition, we found that three fusion genes were differentially expressed between the thyroid cancer and normal tissues, including NTM, SLC7A7 and RXRG. The three genes were all overexpressed in thyroid cancer, with a fusion frequency: NTM-OPCML (16.67\%), RXRG-POU2F1 (5.56\%) and SLC7A7-TRAJ24 (5.56\%) (Fig. 6C and D). Finally, no driver candidate was found to be implicated in fusion genes, indicating that gene fusion may not be the primary mechanism for the involvement of driver genes in thyroid cancer.

\section{Discussion}

In the present study, we carried out a full analysis on the somatic mutations generated by whole exome sequencing of thyroid cancer samples. We found 53 cancer gene candidates and 75 cancer pathways. Among them, BRAF, NRAS, HRAS, EIF1AX, CHEK2 and PPM1D are recurrently mutated (6). $\mathrm{BRAF}$ as a proto-oncogene regulates the MAP kinase/ERK signaling pathway, which affects cell division, differentiation and secretion. Mutations within BRAF contribute to carcinogenesis of a variety of cancer types, such as thyroid cancer (32) and melanoma (33). KRAS, HRAS and NRAS are the most common oncogenes from the RAS gene family. They encode proteins of the GTPase superfamily which plays a great role in signal transduction, protein biosynthesis, cell division, translocation of proteins and transport of vesicles. Mutations in RAS proteins are associated with $\sim 30 \%$ of all human cancers (34). PPM1D is a member of the PP2C family which is known to be a 


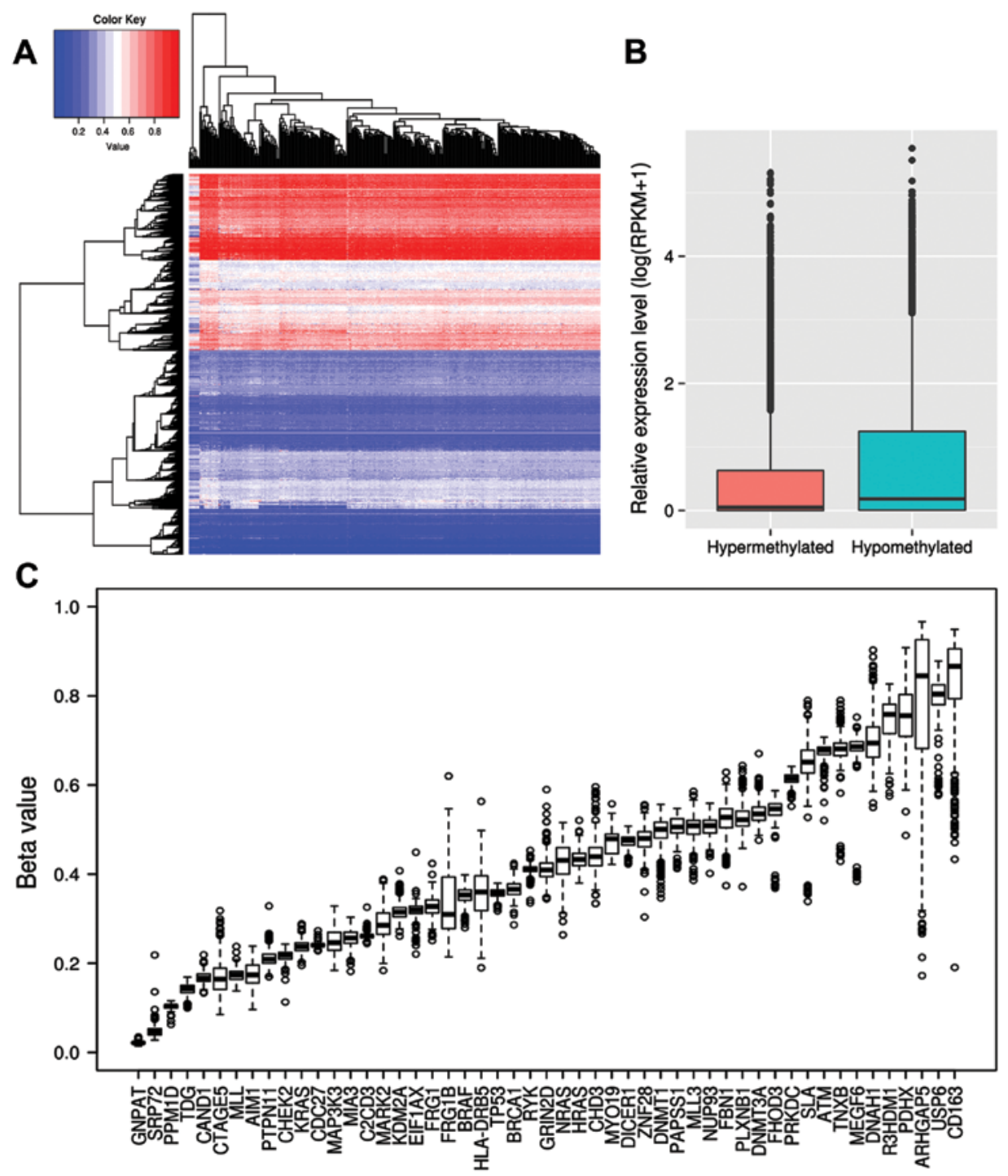

Figure 3. (A) DNA methylation clustering for all protein-coding genes. The average $\beta$ value of DNA methylation was computed for each gene and patient, and undefined values were replaced with the mean. (B) Expression difference between the top 500 hypermethylated and hypomethylated genes. (C) DNA methylation status for all driver candidates. The average $\beta$ value was calculated for each gene and patient.
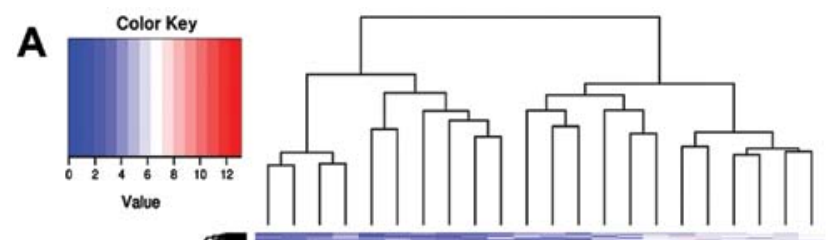

B
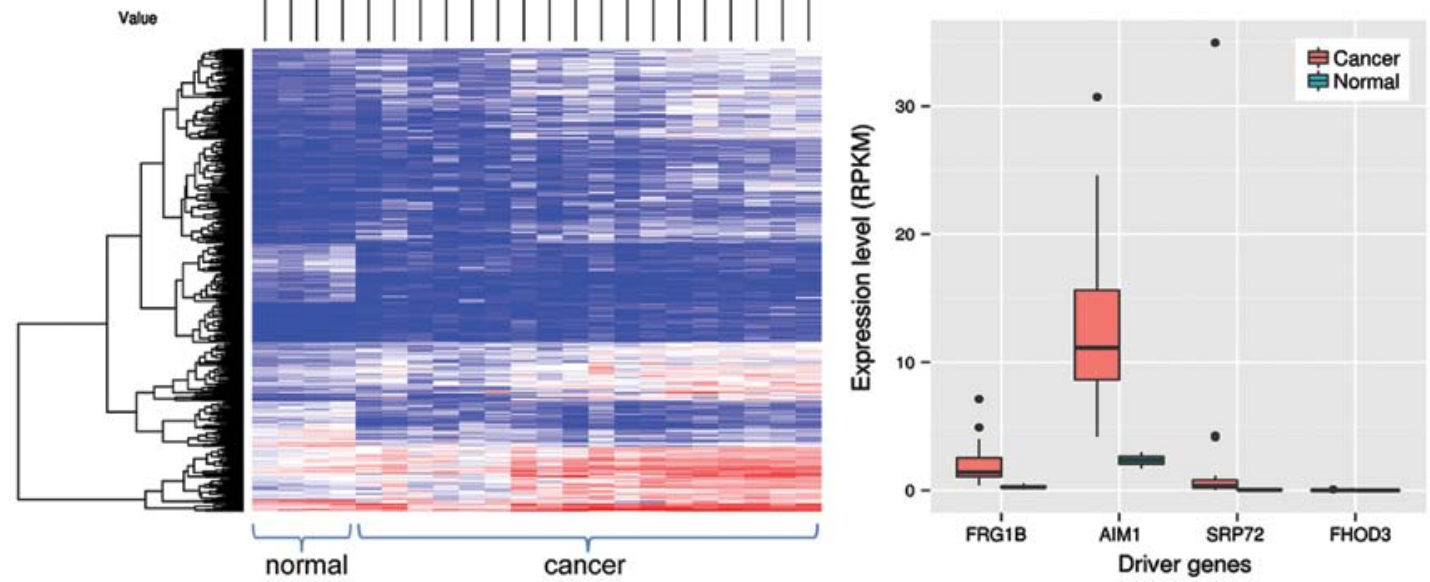

Figure 4. (A) Expression clustering of 504 differentially expressed genes between thyroid cancer and normal samples. The read count of each gene was plus 1 and log scaled. (B) The expression difference of 4 driver candidates between thyroid cancer and normal samples. 


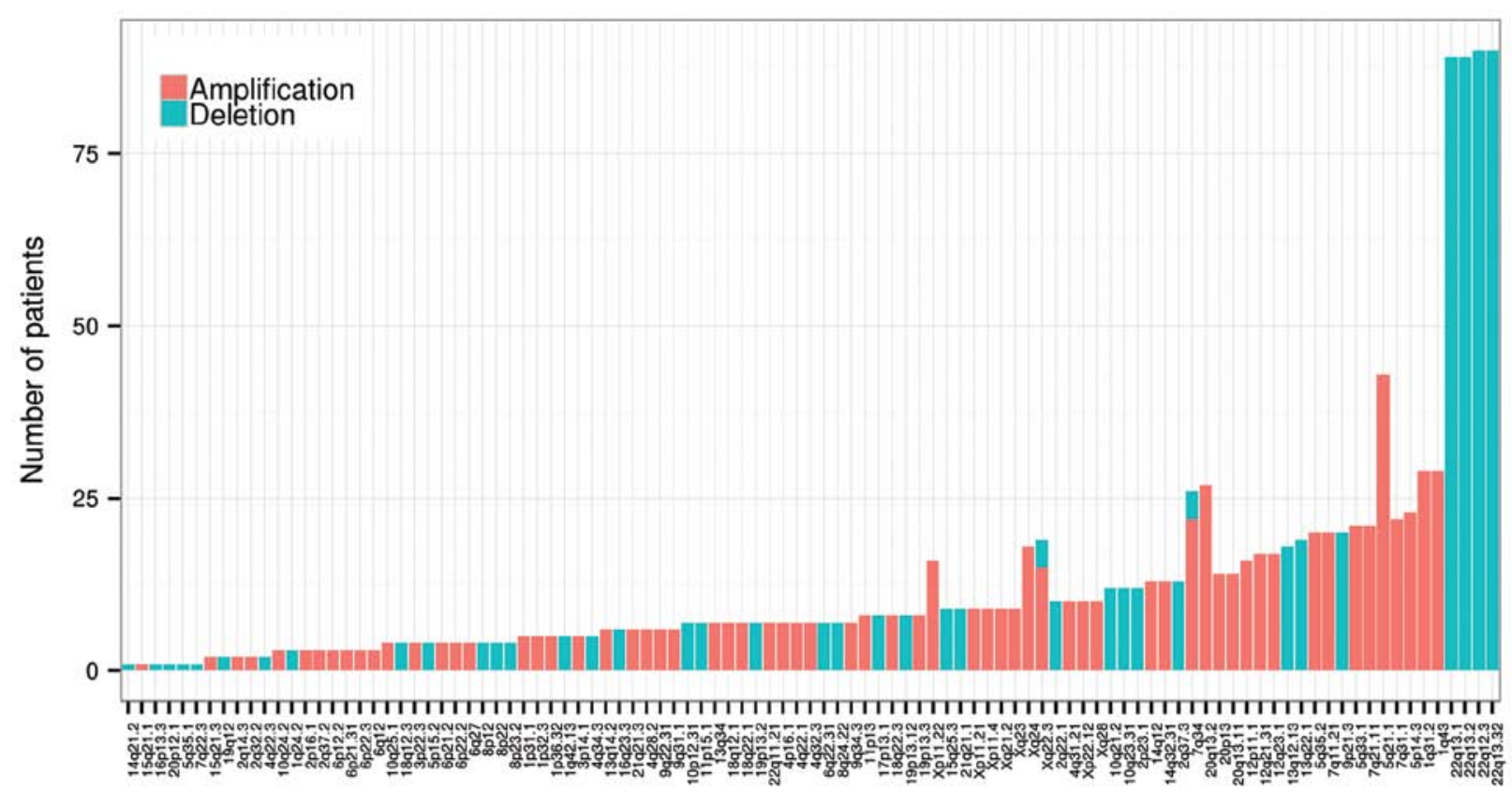

Figure 5. The frequency of copy number variations in 501 thyroid cancer samples.

A

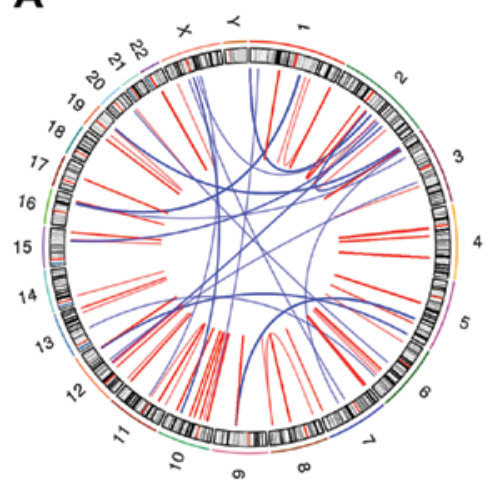

B

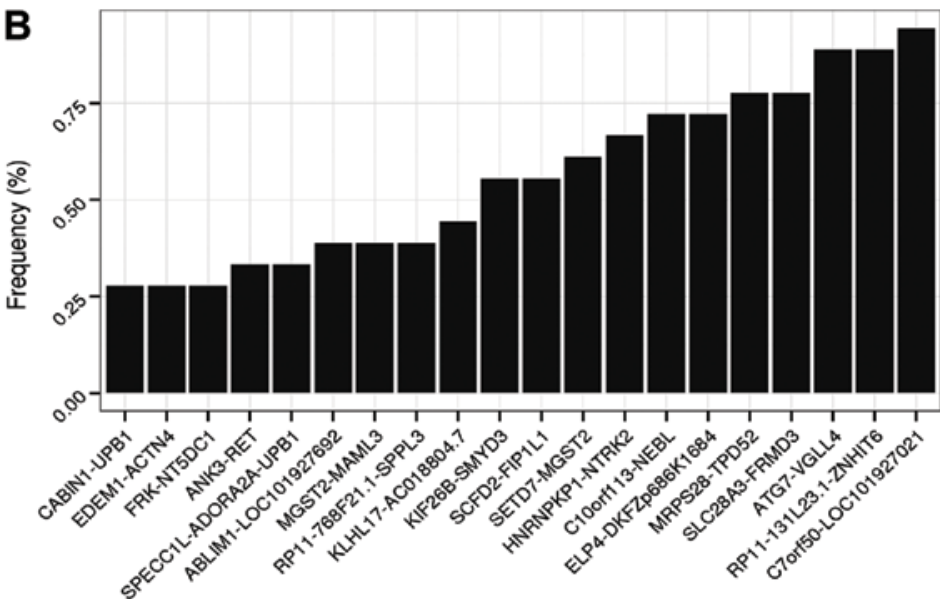

C

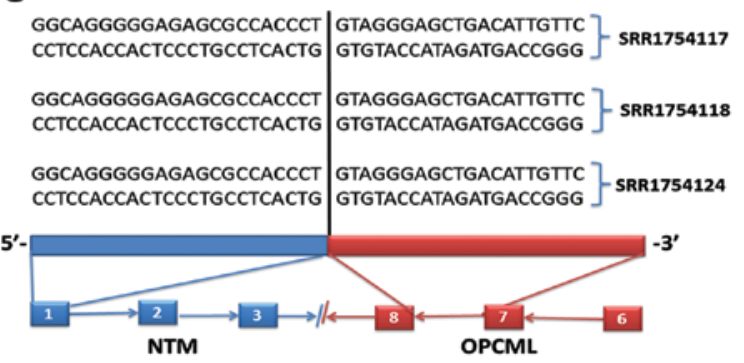

D

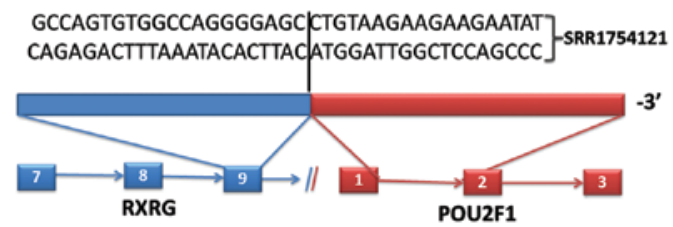

Figure 6. (A) A circus plot shows 91 fusion genes detected by RNA-seq of 18 thyroid cancer samples. The outer ring displays the chromosome ideogram, and pairs of fusion genes are shown with colored arcs which connect the two loci from the same (red) or different chromosomes (blue). (B) The frequency of top 20 pairs of fusion genes in thyroid cancer. (C) NTM-OPCML fusion is between exon 1 of NTM (blue) and intron 7 of OPCML (red) in three thyroid cancer samples. (D) RXRG- POU2F1 fusion is between exon 9 of RXRG (blue) and intron 1 of POU2F1 (red) in one thyroid cancer sample.

negative regulator of cell stress response pathways. It has been reported to be involved in multiple human tumors, such as lung cancer (35), breast cancer and ovarian cancer (36). However, the majority of driver candidates were new cancer genes with low mutation frequency. A great advantage of OncodriveFM and Dendrix is that these two tools identify genes and pathways which accumulate variants of high function impact or mutationally exclusive, independent of the cancer mutation 
frequency. Therefore, application of these tools in cancer research enables us to better explore cancer-driving genes and pathways in the cancer genome. In addition, we also identified many drivers that were differentially expressed, hypermethylated, hypomethylated and CNV-associated, such as SRP72 and FRG1B, suggesting that these genes may contribute to the formation and progression of thyroid cancer in various ways.

We found 91 pairs of fusion genes using RNA-seq data. Three of them, NTM, SLC7A7 and RXRG fusion genes, were overexpressed in thyroid cancer; however, their fusion partners were not affected. These fusion genes are actively implicated in various cancer types, for instance, RXRG is a member of the Retinoid X receptors. It shows tumor-suppressor function in NSCLC (37) and colon cancer (38). RXRG upregulation is associated with dedifferentiation, advanced tumor stage and metastasis (39) and increased apoptosis (40) in thyroid cancer. POU class 2 homeobox 1 (POU2F1), also known as OCT1, is a ubiquitous member of the POU transcription factor family. POU2F1 shows pro-proliferative and pro-apoptotic activity in bladder carcinoma (41). Neurotrimin (NTM) and OPCML are members of the IgLON family of immunoglobulin (Ig) domain-containing glycosylphosphatidylinositol (GPI)-anchored cell adhesion molecules. OPCML as a tumor-suppressor gene is commonly inactivated by either allele loss or DNA methylation in epithelial ovarian cancer (42). SLC7A7 is overexpressed in glioblastoma (GBM), and its overexpression indicates a poor outcome of GBM (43). Genetic variants in SLC7A7 are associated with the risk of glioma (44). Therefore, pairs of these fusion genes may play an important role in the carcinogenesis of thyroid cancer.

In conclusion, taken together, we successfully found a set of cancer-related genes, pathways and fusion genes in thyroid cancer. The findings provide new insight into the pathogenesis of thyroid cancer, therefore paving a potential avenue by which to cure thyroid cancer, based on the disruption of driver genes, pathways and fusion gene pairs.

\section{References}

1. Girardi FM, Barra MB and Zettler CG: Analysis of pattern of occurrence of thyroid carcinoma between 2001 and 2010. Braz J Otorhinolaryngol 81: 541-548, 2015.

2. Hay ID, Thompson GB, Grant CS, Bergstralh EJ, Dvorak CE, Gorman CA, Maurer MS, McIver B, Mullan BP, Oberg AL, et al: Papillary thyroid carcinoma managed at the Mayo Clinic during six decades (1940-1999): Temporal trends in initial therapy and long-term outcome in 2444 consecutively treated patients. World J Surg 26: 879-885, 2002.

3. Forbes SA, Bindal N, Bamford S, Cole C, Kok CY, Beare D, Jia M, Shepherd R, Leung K, Menzies A, et al: COSMIC: Mining complete cancer genomes in the catalogue of somatic mutations in cancer. Nucleic Acids Res 39 (Database): D945-D950, 2011.

4. Lawrence MS, Stojanov P, Polak P, Kryukov GV, Cibulskis K, Sivachenko A,Carter SL, StewartC,MermelCH, Roberts SA, et al: Mutational heterogeneity in cancer and the search for new cancer-associated genes. Nature 499: 214-218, 2013.

5. Dees ND, Zhang Q, Kandoth C, Wendl MC, Schierding W, Koboldt DC, Mooney TB, Callaway MB, Dooling D, Mardis ER, et al: MuSiC: Identifying mutational significance in cancer genomes. Genome Res 22: 1589-1598, 2012.

6. Cancer T and Atlas G; Cancer Genome Atlas Research Network: Integrated genomic characterization of papillary thyroid carcinoma. Cell 159: 676-690, 2014.

7. Wood LD, Parsons DW, Jones S, Lin J, Sjöblom T, Leary RJ, Shen D, Boca SM, Barber T, Ptak J, et al: The genomic landscapes of human breast and colorectal cancers. Science 318 1108-1113, 2007.
8. Gonzalez-Perez A and Lopez-Bigas N: Functional impact bias reveals cancer drivers. Nucleic Acids Res 40: e169, 2012.

9. Ng PC and Henikoff S: SIFT: Predicting amino acid changes that affect protein function. Nucleic Acids Res 31: 3812-3814, 2003.

10. Adzhubei IA, Schmidt S, Peshkin L, Ramensky VE, Gerasimova A, Bork P, Kondrashov AS and Sunyaev SR: A method and server for predicting damaging missense mutations. Nat Methods 7: 248-249, 2010.

11. Reva B, Antipin Y and Sander C: Predicting the functional impact of protein mutations: Application to cancer genomics. Nucleic Acids Res 39: e118, 2011.

12. Vandin F, Upfal E and Raphael BJ: De novo discovery of mutated driver pathways in cancer. Genome Res 22: 375-385, 2012.

13. McLaren W, Pritchard B, Rios D, Chen Y, Flicek P and Cunningham F: Deriving the consequences of genomic variants with the Ensembl API and SNP effect predictor. Bioinformatics 26: 2069-2070, 2010.

14. Ashburner M, Ball CA, Blake JA, Botstein D, Butler $\mathrm{H}$ Cherry JM, Davis AP, Dolinski K, Dwight SS, Eppig JT, et al; The Gene Ontology Consortium: Gene ontology: Tool for the unification of biology. Nat Genet 25: 25-29, 2000.

15. Costa V, Esposito R, Ziviello C, Sepe R, Bim LV, Cacciola NA, Decaussin-Petrucci M, Pallante P, Fusco A and Ciccodicola A: New somatic mutations and WNK1-B4GALNT3 gene fusion in papillary thyroid carcinoma. Oncotarget 6: 11242-11251, 2015.

16. Kim D, Pertea G, Trapnell C, Pimentel H, Kelley R and Salzberg SL: TopHat2: Accurate alignment of transcriptomes in the presence of insertions, deletions and gene fusions. Genome Biol 14: R36, 2013.

17. Quinlan AR and Hall IM: BEDTools: A flexible suite of utilities for comparing genomic features. Bioinformatics 26: 841-842, 2010.

18. Love MI, Huber W and Anders S: Moderated estimation of fold change and dispersion for RNA-seq data with DESeq2. Genome Biol 15: 550, 2014

19. Lawrence MS, Stojanov P, Mermel CH, Robinson JT, Garraway LA, Golub TR, Meyerson M, Gabriel SB, Lander ES and Getz G: Discovery and saturation analysis of cancer genes across 21 tumour types. Nature 505: 495-501, 2014.

20. Zhong Q, Peng H-L, Zhao X, Zhang L and Hwang W-T: Effects of BRCA $1 / 2$ on ovarian and breast cancer survival-response. Clin Cancer Res 21: 3807-3807, 2015.

21. Fujimoto A, Totoki Y, Abe T, Boroevich KA, Hosoda F, Nguyen HH, Aoki M, Hosono N, Kubo M, Miya F, et al: Whole-genome sequencing of liver cancers identifies etiological influences on mutation patterns and recurrent mutations in chromatin regulators. Nat Genet 44: 760-764, 2012.

22. Zang ZJ, Cutcutache I, Poon SL, Zhang SL, McPherson JR, Tao J, Rajasegaran V, Heng HL, Deng N, Gan A, et al: Exome sequencing of gastric adenocarcinoma identifies recurrent somatic mutations in cell adhesion and chromatin remodeling genes. Nat Genet 44: 570-574, 2012.

23. Golding SE, Rosenberg E, Adams BR, Wignarajah S, Beckta JM, O'Connor MJ and Valerie K: Dynamic inhibition of ATM kinase provides a strategy for glioblastoma multiforme radiosensitization and growth control. Cell Cycle 11: 1167-1173, 2012.

24. Negi SS and Brown P: rRNA synthesis inhibitor, CX-5461, activates ATM/ATR pathway in acute lymphoblastic leukemia, arrests cells in G2 phase and induces apoptosis. Oncotarget 6: 18094-18104, 2015.

25. Bentires-Alj M, Paez JG, David FS, Keilhack $\mathrm{H}$, Halmos B, Naoki K, Maris JM, Richardson A, Bardelli A, Sugarbaker DJ, et al: Activating mutations of the noonan syndrome-associated SHP2/PTPN11 gene in human solid tumors and adult acute myelogenous leukemia. Cancer Res 64: 8816-8820, 2004.

26. Seki M, Yoshida K, Shiraishi Y, Shimamura T, Sato Y, Nishimura R, Okuno Y, Chiba K, Tanaka H, Kato K, et al: Biallelic DICER1 mutations in sporadic pleuropulmonary blastoma. Cancer Res 74: 2742-2749, 2014.

27. Hasan R, Sharma R, Saraya A, Chattopadhyay TK, DattaGupta S, Walfish PG, Chauhan SS and Ralhan R: Mitogen activated protein kinase kinase kinase 3 (MAP3K3/MEKK3) overexpression is an early event in esophageal tumorigenesis and is a predictor of poor disease prognosis. BMC Cancer 14: 2, 2014.

28. Samanta AK, Huang HJ, Bast RC Jr and Liao WS-L: Overexpression of MEKK3 confers resistance to apoptosis through activation of NFkappaB. J Biol Chem 279: 7576-7583, 2004. 
29. Arndt S and Bosserhoff AK: Reduced expression of TANGO in colon and hepatocellular carcinomas. Oncol Rep 18: 885-891, 2007.

30. Arndt $\mathrm{S}$ and Bosserhoff AK: TANGO is a tumor suppressor of malignant melanoma. Int J Cancer 119: 2812-2820, 2006.

31. Gonzalo S: Epigenetic alterations in aging. J Appl Physiol 1985 109: 586-597, 2010.

32. Xing M, Alzahrani AS, Carson KA, Shong YK, Kim TY, Viola D, Elisei R, Bendlová B, Yip L, Mian C, et al: Association between BRAF V600E mutation and recurrence of papillary thyroid cancer. J Clin Oncol 33: 42-50, 2015.

33. Inumaru JS, Gordo KI, Fraga Junior AC, Silva AM, Leal CB, Ayres FM, Wastowski IJ, Borges NF and Saddi VA: Analysis of the BRAF V600E mutation in primary cutaneous melanoma. Genet Mol Res 13: 2840-2848, 2014.

34. Parikh C, Subrahmanyam R and Ren R: Oncogenic NRAS, KRAS, and HRAS exhibit different leukemogenic potentials in mice. Cancer Res 67: 7139-7146, 2007.

35. Zhang C, Chen Y, Wang M, Chen X, Li Y, Song E, Liu X, Kim S and Peng H: PPM1D silencing by RNA interference inhibits the proliferation of lung cancer cells. World J Surg Oncol 12: 258, 2014.

36. Ruark E, Snape K, Humburg P, Loveday C, Bajrami I, Brough R, Rodrigues DN, Renwick A, Seal S, Ramsay E, et al; Breast and Ovarian Cancer Susceptibility Collaboration; Wellcome Trust Case Control Consortium: Mosaic PPM1D mutations are associated with predisposition to breast and ovarian cancer. Nature 93: 406-410, 2013.

37. Brabender J, Danenberg KD, Metzger R, Schneider PM, Lord RV Groshen S, Tsao-Wei DD, Park J, Salonga D, Hölscher AH, et al: The role of retinoid $\mathrm{X}$ receptor messenger RNA expression in curatively resected non-small cell lung cancer. Clin Cancer Res 8: 438-443, 2002.
38. Papi A, Rocchi P, Ferreri AM and Orlandi M: RXRgamma and PPARgamma ligands in combination to inhibit proliferation and invasiveness in colon cancer cells. Cancer Lett 297: 65-74, 2010.

39. Liu Z, Zhou G, Nakamura M, Bai Y, Li Y, Ozaki T, Mori I, Miyauchi A and Kakudo K: Retinoid X receptor $\gamma$ up-regulation is correlated with dedifferentiation of tumor cells and lymph node metastasis in papillary thyroid carcinoma. Pathol Int 61: 109-115, 2011.

40. Klopper JP, Hays WR, Sharma V, Baumbusch MA, Hershman JM and Haugen BR: Retinoid X receptor-gamma and peroxisome proliferator-activated receptor-gamma expression predicts thyroid carcinoma cell response to retinoid and thiazolidinedione treatment. Mol Cancer Ther 3: 1011-1020, 2004.

41. Szekeres K, Koul R, Mauro J, Lloyd M, Johnson J and Blanck G: An Oct-1-based, feed-forward mechanism of apoptosis inhibited by co-culture with Raji B-cells: Towards a model of the cancer cell/B-cell microenvironment. Exp Mol Pathol 97: 585-589, 2014.

42. Sellar GC, Watt KP, Rabiasz GJ, Stronach EA, Li L, Miller EP, Massie CE, Miller J, Contreras-Moreira B, Scott D, et al: OPCML at $11 \mathrm{q} 25$ is epigenetically inactivated and has tumor-suppressor function in epithelial ovarian cancer. Nat Genet 34: 337-343, 2003.

43. Fan S, Meng D, Xu T, Chen Y, Wang J, Li X, Chen H, Lu D, Chen J and Lan Q: Overexpression of SLC7A7 predicts poor progression-free and overall survival in patients with glioblastoma. Med Oncol 30: 384, 2013.

44. Fan S, Zhao Y, Li X, Du Y, Wang J, Song X, Zhou F, Chen H, Chen G, Zhao Y, et al: Genetic variants in SLC7A7 are associated with risk of glioma in a Chinese population. Exp Biol Med (Maywood) 238: 1075-1081, 2013. 